\title{
A Novel Orthohepadnavirus Identified in a Dead Maxwell's Duiker (Philantomba maxwellii) in Taï National Park, Côte d'Ivoire
}

\author{
Jan F. Gogarten ${ }^{1,2,3,4} \oplus$, Markus Ulrich ${ }^{1}$, Nishit Bhuva ${ }^{3}$, Joel Garcia ${ }^{3}$, Komal Jain ${ }^{3}$, \\ Bohyun Lee ${ }^{3}$, Therese Löhrich ${ }^{1,5}$, Alexandra Oleynik ${ }^{3}$, Emmanuel Couacy-Hymann ${ }^{6}$, \\ Terence Fuh Neba ${ }^{5}$, Nischay Mishra ${ }^{3}$, Thomas Briese ${ }^{3, *,+}{ }^{4}$, Sébastien Calvignac-Spencer ${ }^{1,2, *,+}$, \\ W. Ian Lipkin $3, *,+$ and Fabian H. Leendertz ${ }^{1,2, *,+}$ \\ 1 Epidemiology of Highly Pathogenic Microorganisms, Robert Koch Institute, 13353 Berlin, Germany; \\ jan.gogarten@gmail.com (J.F.G.); UlrichM@rki.de (M.U.); tloehrich@wwfcar.org (T.L.) \\ 2 Viral Evolution, Robert Koch Institute, 13353 Berlin, Germany \\ 3 Center for Infection and Immunity, Mailman School of Public Health, Columbia University, \\ New York, NY 10027, USA; npb2123@cumc.columbia.edu (N.B.); jg2876@cumc.columbia.edu (J.G.); \\ kj2230@cumc.columbia.edu (K.J.); bl2493@cumc.columbia.edu (B.L.); ap2811@cumc.columbia.edu (A.O.); \\ nm2641@cumc.columbia.edu (N.M.) \\ 4 Institute of Microbiology and Epizootics, Freie Universität Berlin, 14163 Berlin, Germany \\ 5 World Wide Fund for Nature, Dzanga-Sangha Protected Areas, Bangui BP 1053, Central African Republic; \\ TNeba@wwfcar.org \\ 6 Laboratoire National D'appui au Développement Agricole/Laboratoire Central de Pathologie Animale, \\ Bingerville BP206, Côte d'Ivoire; chymann@hotmail.com \\ * Correspondence: tb2047@cumc.columbia.edu (T.B.); CalvignacS@rki.de (S.C.-S.); \\ wil2001@cumc.columbia.edu (W.I.L.); LeendertzF@rki.de (F.H.L.) \\ + These authors contributed equally to the manuscript.
}

Received: 28 February 2019; Accepted: 16 March 2019; Published: 19 March 2019

Abstract: New technologies enable viral discovery in a diversity of hosts, providing insights into viral evolution. We used one such approach, the virome capture sequencing for vertebrate viruses (VirCapSeq-VERT) platform, on 21 samples originating from six dead Maxwell's duikers (Philantomba maxwellii) from Taï National Park, Côte d'Ivoire. We detected the presence of an orthohepadnavirus in one animal and characterized its $3128 \mathrm{bp}$ genome. The highest viral copy numbers were detected in the spleen, followed by the lung, blood, and liver, with the lowest copy numbers in the kidney and heart; the virus was not detected in the jejunum. Viral copy numbers in the blood were in the range known from humans with active chronic infections leading to liver histolytic damage, suggesting this virus could be pathogenic in duikers, though many orthohepadnaviruses appear to be apathogenic in other hosts, precluding a formal test of this hypothesis. The virus was not detected in 29 other dead duiker samples from the Côte d'Ivoire and Central African Republic, suggesting either a spillover event or a low prevalence in these populations. Phylogenetic analysis placed the virus as a divergent member of the mammalian clade of orthohepadnaviruses, though its relationship to other orthohepadnaviruses remains uncertain. This represents the first orthohepadnavirus described in an artiodactyl. We have tentatively named this new member of the genus Orthohepadnavirus (family Hepadnaviridae), Taï Forest hepadnavirus. Further studies are needed to determine whether it, or some close relatives, are present in a broader range of artiodactyls, including livestock.

Keywords: virus; hepadnavirus; Orthohepadnavirus; discovery; hybridization capture; bovid; artiodactyls; sequencing 


\section{Introduction}

Partially double stranded DNA viruses belonging to the genus Orthohepadnavirus infect a number of species of nonhuman primates [1-4], bats [5-8], rodents [9-12], and the domestic cat [13]. The type species of the genus, Hepatitis B virus (HBV), remains a major public health concern in humans; in 2015 there were an estimated 257 million people with chronic HBV infections resulting in an estimated 887,000 deaths [14]. In animal host species, infection with orthohepadnaviruses results in signs ranging from hepatitis, to hematomas, and hepatocellular carcinomas (e.g., in woodchucks (Marmota monax) [9], arctic ground squirrels (Spermophylus parryi kennicotti) [10], and woolly monkey (Lagothrix lagotricha) [1]). In vitro studies showed that bat hepatitis viruses have the potential to infect human hepatocytes, highlighting the zoonotic potential of orthohepadnaviruses [15]. Similarly, recombination events between human and primate $\mathrm{HBV}$, and detection of likely transmission events between humans and chimpanzees [16], highlight the potential for cross-species transmission and the importance of understanding orthohepadnavirus ecology and evolution in wildlife [17]. Here, we report the discovery of a novel orthohepadnavirus in a deceased wild Maxwell's duiker (Philantomba maxwellii) in Taï National Park, Côte d'Ivoire. The discovery of this virus in a bovid represents the first indication of hepadnaviruses in artiodactyls, expanding the potential host range of these viruses to a mammalian order that includes many important livestock species.

\section{Methods and Results}

In the course of a long-term research project on wildlife mortality in Taï National Park, Côte d'Ivoire and Dzanga-Sangha Protected Areas (DSPA), Central African Republic, necropsy samples were systematically collected by on site veterinarians and preserved for analyses [18-20]. The carcass positive for the novel orthohepadnavirus, was found by field assistants working with the Taï Chimpanzee Project in January 2015 (GPS coordinates: latitude: 5.854, longitude: -7.299). The carcass was found in the core research area of the Taï Chimpanzee Project, where human hunting and trapping activities are rare. We found no evidence on the carcass suggestive of human involvement in the death of this animal, nor evidence of external lesions suggestive of trauma prior to death. The veterinarian on site performed a necropsy on the day after the carcass was found. The liver of this animal was swollen and parts of jejunum and duodenum appeared to be hemorrhagically infiltrated, though maggots were already present on and in the carcass, and advanced autolysis precluded a detailed histopathological description and made an assessment of the degree of swelling and hemorrhage difficult. Samples of heart blood, heart, duodenum, jejunum, kidney, liver, lung, and spleen were collected, fixed in formalin or stored fresh in liquid nitrogen and transported on dry ice to the Robert Koch Institute, Berlin, Germany where they were stored at $-80^{\circ} \mathrm{C}$ until further analysis was performed. Initially, 21 samples from 6 duikers were analyzed (Table S1) that were negative for other known wildlife pathogens that cause mortality in this ecosystem (i.e., orthopoxviruses [21], Bacillus cereus biovar anthracis [20]; tested using the diagnostic systems described in [22] and [20], respectively).

Nucleic acids were extracted; frozen tissue samples were using the QIAamp Viral RNA mini Kit (Qiagen, Hilden, Germany) according to the manufacturer's instructions, but modified the protocol by homogenizing samples with $280 \mu \mathrm{L}$ AVL buffer and 20-30 ceramic beads in a Fastprep-24 homogenizer ( $4 \mathrm{~m} / \mathrm{s}$ for $30 \mathrm{~s}$ followed by a cooling step for $2 \mathrm{~min}$ on dry ice, for a total of 3 homogenization cycles: MP Biomedicals). By not using RNase, this kit extracts total nucleic acids (TNA). Extractions were also performed without carrier RNA. On the extracted nucleic acid, we performed high throughput sequencing using the virome capture sequencing for vertebrate viruses (VirCapSeq-VERT) platform [23]. For each batch of libraries that were pooled for capture, we also processed salmon sperm DNA as negative control (Table S1). Briefly, TNA was reverse transcribed using SuperScript IV (Thermo Fisher, Waltham, MA, USA) with random hexamers, and the cDNA was RNase H treated prior to second-strand synthesis with Klenow fragment (New England Biolabs, Ipswich, MA, USA). The resulting double-stranded cDNA/DNA mix was sheared by ultrasonication to an average 
fragment size of $200 \mathrm{bp}$ following manufacturer's instructions (Covaris E210 focused ultrasonicator). Sheared product was purified using AxyPrep beads (Axygen, Union City, CA, USA) and libraries were prepared with KAPA Hyper Prep kits (KAPA) and custom unique dual barcoding indices. Library quality and quantity was assessed using a Bioanalyzer (Agilent, Santa Clara, CA, USA), before hybridization capture with VirCapSeq-VERT probes [23] and sequencing on an Illumina HiSeq 2500 system. Sequenced reads were demultiplexed with cutadapt (v1.15) and quality filtered and trimming with PRINSEQ software (v 0.20.3). Reads were filtered if the average quality score of a read was less than thirty, if the minimum length was less than 50, and if the entropy was less than 70 . The first nine bases and the last base of reads were further trimmed. The full Maxwell's duiker genome has not been sequenced; therefore, we used a Bos taurus genome (GCF_000003055.6) for host subtraction; a Bowtie index was created for the host genome and mapping of the filtered reads was performed using bowtie 2 mapper with default settings. Reads that matched the host genome at $\geq 90 \%$ identity were discarded. Host subtracted reads were then assembled using Mira (v 4.0) with SOLEXA settings, with a minimum overlap of 20 between the reads and a minimum of three reads per contig. Contigs and singletons were then collapsed using CD-HIT at $100 \%$ identity and subjected to homology search by MegaBlast against the GenBank nucleotide database and sequences not assigned at the nucleotide level were screened by BLASTx to detect divergent or potentially new viruses. Based on the BLAST results, contigs and singletons were assigned at family, genus, species, and GenBank accession number level to identify the most closely related GenBank matches. Hepadnavirus sequences were found in the liver and duodenum of the same animal (Table 1). We detected no other viruses in the liver or duodenum of this animal using VirCapSeq-Vert.

Table 1. Read processing of samples containing hits to a orthohepadnavirus.

\begin{tabular}{ccccc}
\hline Tissue & Total Reads & $\begin{array}{c}\text { Reads after } \\
\text { Filtering }\end{array}$ & $\begin{array}{c}\text { Reads after Host } \\
\text { Subtraction }\end{array}$ & $\begin{array}{c}\text { Reads with Top Blast Hit } \\
\text { to a Orthohepadnavirus }\end{array}$ \\
\hline Liver & $10,548,728$ & $10,111,516$ & $9,105,066$ & $2,475,043$ \\
Duodenum & $9,776,183$ & $9,082,857$ & $6,249,455$ & 964 \\
\hline
\end{tabular}

To assemble the full genome of the detected virus, tentatively named Taï Forest hepadnavirus, we downsampled to 1,000,000 reads with SEQTK to make subsequent bioinformatics steps more tractable (https: / / github.com/lh3/seqtk). Reads were trimmed using Trimmomatic v0.38, removing the leading and trailing reads below Q30, and clipping any part of the read where the average base quality across $4 \mathrm{bp}$ was less than 30 [24]. Nineteen contigs longer than $500 \mathrm{nt}$ were assembled using Spades and default settings [25]. BLAST alignment against the NCBI database indicated that two contigs were hits to orthohepadnavirus sequences. These contigs did not overlap, so we extended them by iteratively mapping our trimmed reads against these contigs with Geneious v11.1.5 [26] using standard settings (5 iterations: http:/ / www.geneious.com). We generated a majority rule consensus sequence for each of those two contigs, requiring a minimum of $10 \times$ coverage for a base to be called. This resulted in two contigs, one of $2026 \mathrm{nt}$ that did not contain a poly-A tail, and another, that included a poly-A tail towards the end, with $134 \mathrm{bp}$ following the poly-A tail. We removed the poly-A tail and the $134 \mathrm{nt}$ following it, and ran a de novo assembly of the two contigs, resulting in an overlap of $191 \mathrm{nt}$ We split the combined long contig into two parts and re-aligned them with Geneious using default settings, which resulted in an overlap with 100\% identity, suggesting that we had sequenced a $3128 \mathrm{nt}$ circular genomic sequence.

After trimming and quality filtering as above, we mapped the entire read set obtained for the positive duiker sample to this circular genome using BWA [27], resulting in 3,569,327 reads mapping from the positive liver sample and 1330 reads from the duodenum sample (deduplicated $=633,930$ and 326 unique reads, respectively; Table S1). This represented 100\% genome coverage at a minimum depth of $10 \times$ for the liver sample and $10.2 \%$ and $38.5 \%$ genome coverage at $10 \times$ and $1 \times$, respectively, for the duodenum sample. As shown in Table S1, in sample pools with highly positive specimens 
we encountered background noise, which in batch 2 amounted to 11 to 72 reads (or $0.001-0.007 \%$ of the highest signal, when normalized to 10 million total reads generated). This background was not considered as biologically meaningful as it stayed below our positivity cut-off of $0.015 \%$ of the highest signal, or not more than $3 \times$ the read count detected in the negative salmon sperm control that ran in that pool (Table S1).

We annotated the circular genome generated from the liver sample in Geneious based on similarity to the long-fingered bat hepatitis B virus (NC_020881) and human hepatitis B virus (NC_003977) by using a $50 \%$ identity threshold to transfer reference annotations (Figure $1 \mathrm{~A}$ ). We also examined the Pfam alignments (https://pfam.xfam.org). Whereas $\mathrm{P}, \mathrm{X}$, and PreC/C open reading frame (ORF) sequences were readily recognizable, highly divergent sequence between the preS1 and S ORFs obscure the potential start position of preS2 sequence. Two 11-nt direct repeats located at the start of the preC and the end of the P coding sequence (DR1 and DR2, TTCACCTgTGC) were well conserved when compared to repeats found in the same positions in hepatitis B viruses (TTCACCTcTGC).

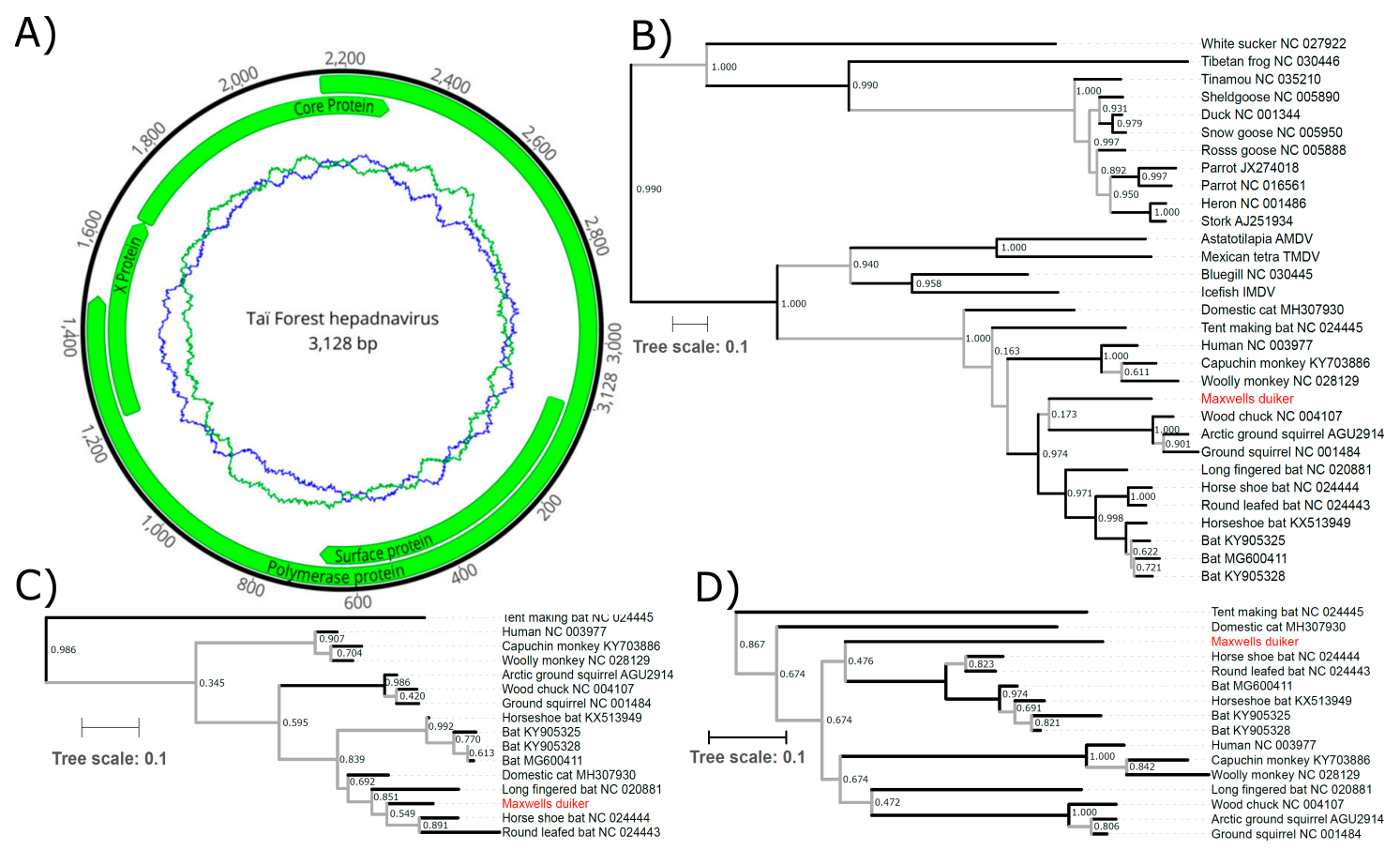

Figure 1. (A) The genome organization of Taï Forest hepadnavirus. The innermost circles represent the GC (blue) and AT (green) content along a 50 nt sliding window. (B) Phylogenetic relationship of Taï Forest hepadnavirus to other viruses of the family Hepadnaviridae. Maximum likelihood phylogeny constructed using the amino acid sequence of the polymerase ORF from a range of vertebrate hepadnaviruses; and (C) using the core and (D) the surface protein ORFs in comparison to the mammalian orthohepadnaviruses; the common name of the host is indicated at the branch labels, along with each virus's accession number. GenBank sequences were aligned to Taï Forest hepadnavirus ORF sequences using MAFFT (v7.307) and we selected conserved blocks using Gblocks, as implemented in SeaView V4 [28]. PhyML with smart model selection [29], and the Bayesian Information Criterion and subtree pruning and regrafting (SPR) was applied as the tree improvement approach, with otherwise default settings for tree building (selected models: polymerase ORF $=\mathrm{LG}+\mathrm{G}+\mathrm{I}+\mathrm{F}$; core ORF $=\mathrm{JTT}+$ $\mathrm{G}$; surface ORF $=\mathrm{JTT}+\mathrm{G}+\mathrm{F}$ ). We estimated the best-fitting root of these phylogenies using the heuristic residual mean squared function in the program TempEst, which minimizes the variance of root-to-tip distances [30]. To further assess the confidence in our phylogenetic trees, BMCMC analyses were run on each amino acid alignment using BEAST v1.10.4 under the assumption of a relaxed log-normal molecular clock and with tree shape modeled according to a birth-death speciation model and the amino acids substitution model supported by PhyML's smart model selection [31]. We examined the 
output of three runs for convergence and appropriate sampling of the posterior using Tracer v1.7.1 [32] before merging runs using LogCombiner v1.10.4 [33]. The best representative tree was then identified from the posterior set of trees and annotated with TreeAnnotator v1.10.4 (distributed with BEAST). Branch support was assessed using Shimodaira-Hasegawa-like approximate likelihood ratio tests (SH-like aLRT), with branches supported by SH-like aLRT values $<0.95$ and/or posterior probabilities $<0.95$ in the Bayesian Markov chain Monte Carlo tree indicated in gray. Branch lengths are representative of substitutions per site. SH-like aLRT values are indicated at each node.

To confirm the presence of the virus in these and other duiker samples, we designed four PCR systems in conserved regions of the genome (Table 2) identified through alignment with other mammalian orthohepadnavirus genomes using MAFFT [34]. In addition, we tested the HBVS3s and HBVS3as primers developed by Schaefer, et al. [35]. All PCR systems were tested on the liver sample in a total volume of $25 \mu \mathrm{L}$ containing $0.2 \mathrm{mM}$ dNTP (with dUTP replacing dTTP), $4 \mathrm{mM} \mathrm{MgCl}_{2}, 0.2 \mu \mathrm{M}$ of each primer, 1.25U Platinum ${ }^{\circledR}$ Taq Polymerase (Invitrogen, Waltham, MA, USA), $10 \times$ PCR Buffer (Invitrogen) and PCR-grade water. Each reaction was seeded with $5 \mu \mathrm{L}$ TNA and cycling conditions were: $95^{\circ} \mathrm{C}$ for $5 \mathrm{~min}, 35$ cycles of $\left[95^{\circ} \mathrm{C}\right.$ for $30 \mathrm{~s}$, annealing temp for $30 \mathrm{~s}$ (Table 2), $72{ }^{\circ} \mathrm{C}$ for $1 \mathrm{~min}$ ], followed by a final extension at $72{ }^{\circ} \mathrm{C}$ for $7 \mathrm{~min}$. All primer sets generated amplification products, which were visualized on agarose gels (data not shown). Amplification products were cleaned using ExoSAP-IT $^{\circledR}$ (Affymetrix, Santa Clara, CA, USA). Sequencing reactions were performed with the primers used for amplification and the BigDye Terminator v3.1 cycle kit (Thermo Fisher, Waltham, MA, USA) and Sanger sequenced in both directions with the ABI PRISM ${ }^{\circledR} 3100$ Genetic Analyzer (Applied Biosystems, Waltham, MA, USA). Chromatograms were evaluated using Geneious and were $100 \%$ matches to the genome generated by VirCapSeq-VERT.

Table 2. Primers designed for sequencing and quantification of Taï Forest hepadnavirus.

\begin{tabular}{ccc}
\hline Primer Name & Sequence $\left(5^{\prime}->\mathbf{3}^{\prime}\right)$ & Annealing Temperature $\left({ }^{\circ} \mathbf{C}\right)$ \\
\hline $\begin{array}{c}\text { Orthohep_1F } \\
\text { Orthohep_1R }\end{array}$ & $\begin{array}{c}\text { TGGTGGACTTCTCTCAGTTTTCC } \\
\text { TGATAAAACGCCGCAGACAC }\end{array}$ & 56 \\
\hline $\begin{array}{c}\text { Orthohep_1F } \\
\text { Orthohep_1Rb }\end{array}$ & $\begin{array}{l}\text { TGGTGGACTTCTCTCAGTTTTCC } \\
\text { AGATGAGGCATAGAACCAGGA }\end{array}$ & 57 \\
\hline $\begin{array}{c}\text { Orthohep_2F } \\
\text { Orthohep_2R }\end{array}$ & $\begin{array}{l}\text { TGCTTAGCCATCCCTTCGTCA } \\
\text { GGCCCCCAGTACCACATCAT }\end{array}$ \\
\hline $\begin{array}{c}\text { Orthohep_4F } \\
\text { Orthohep_4R }\end{array}$ & $\begin{array}{l}\text { CACAGGTGAAGCGAAGGACA } \\
\text { CCCCAAWACCAVATCATCCATATA }\end{array}$ & 58 \\
\hline
\end{tabular}

A real-time assay was tested for each primer set, using a reaction volume of $12.5 \mu \mathrm{L}$ containing $6.25 \mu \mathrm{L}$ GoTaq $^{\circledR}$ qPCR Master Mix (Promega, Fitchburg, WI, USA), $0.2 \mu \mathrm{M}$ of each primer and $1 \mu \mathrm{L}$ TNA extract of the positive duiker liver or $1 \mu \mathrm{L}$ diluted PCR product. Standards were generated by serial dilution of PCR products. Cycling conditions were $95^{\circ} \mathrm{C}$ for $5 \mathrm{~min}, 40$ cycles $\left[95^{\circ} \mathrm{C}\right.$ for $15 \mathrm{~s}$, annealing temp (Table S1) for $1 \mathrm{~min}$. Quantitative PCRs (qPCRs) were run on a BioRad CFX96 measuring fluorescence after each extension phase. Results were analyzed using BioRad Maestro software. Based on these results, the most sensitive of the primer sets (Orthohep_2F/Orthohep_2R) was chosen to measure viral copy numbers in all available tissues from the infected duiker to assess viral organ distribution (Table 3). The observed viral copy numbers in the blood were in the range of that found in humans with an active chronic infection that leads to liver damage [36]. This potentially implicates the virus in the duiker's death, although further studies are needed to understand the impact of Taï Forest hepadnavirus on duikers, as many hepadnaviruses appear to be apathogenic in other mammalian hosts [11], precluding a formal test of this hypothesis. In this duiker we did not detect other pathogens that can cause duiker mortality in this ecosystem (i.e., orthopoxviruses [21], Bacillus cereus biovar anthracis [20]; filoviruses [37]), though it is certainly possible that the duiker died 
of causes unrelated to this Taï Forest hepadnavirus. The highest copy numbers were detected in the spleen and blood, and viral copies were detected in all other tissues tested except for the jejunum (Table 3). Orthohepadnavirus replication outside of the liver in lymphoid cells in the spleen has been proposed in woodchucks [38,39], but understanding the tropism of Tai Forest hepadnavirus in more animals and the determinants of orthohepadnaviruses more generally remain important areas of future research. We also used this qPCR to test samples of 23 additional dead duikers found in the Côte d'Ivoire and the Central African Republic (Table S2; collected in areas where human hunting and trapping is rare and with no indication of anthropogenic causes of mortality), alongside the samples previously assessed by VirCapSeq-VERT. We detected no additional positive samples, confirming the negative results found in the VirCapSeq-VERT experiment.

Table 3. Estimated viral copies per duiker genome. To estimate the size of the Maxwell's duiker genome we calculated the average genome size for bovids in the Animal Genome Size Database, Release 2.0 (www.genomesize.com), using the average species genome size for sheep (Ovis aries) and domestic cattle (Bos taurus), for which there were multiple entries. A haploid bovid genome was estimated to roughly equal $3.7 \mathrm{pg}$, so that one Maxwell's duiker cell would contain approximately 7.4 pg of DNA.

\begin{tabular}{ccccc}
\hline Tissue & $\begin{array}{c}\text { Extract Conc. } \\
(\mathbf{n g} / \boldsymbol{\mu L})\end{array}$ & $\begin{array}{c}\text { Duiker } \\
\text { Genomes/ } \boldsymbol{\mu L}\end{array}$ & Viral Copies/ $\boldsymbol{\mu L}$ & $\begin{array}{c}\text { Viral Copies/Duiker } \\
\text { Genome }\end{array}$ \\
\hline Heart blood & 5.9 & 801.4 & 4019.4 & 5.0 \\
Heart & 3.9 & 520.3 & 831.4 & 1.6 \\
Liver & 7.8 & 1059.5 & 3205.0 & 3.0 \\
Spleen & 2.2 & 290.5 & $50,203.6$ & 172.8 \\
Lung & 1.8 & 243.2 & 4903.6 & 20.2 \\
Kidney & 9.1 & 1232.4 & 283.8 & 0.2 \\
Duodenum & 17.7 & 2391.9 & 939.1 & 0.4 \\
Jejunum & 12.0 & 1621.6 & 0.0 & 0.0 \\
\hline
\end{tabular}

As with other hepadnaviruses, the reconstruction of phylogenetic relationships is not straightforward given the diverse results obtained for various coding regions and analyses. We examined the $\mathrm{P}, \mathrm{C}$ and $\mathrm{S}$ amino acid sequences to assess the phylogenetic relationship of Taï Forest hepadnavirus to other representative members of the family Hepadnaviridae. Maximum likelihood trees were constructed, including viruses from both genera, Avihepadnavirus and Orthohepadnavirus, as well as unassigned hepadnaviruses were constructed (Figure 1B). For the shorter, more diverse sequences of the $C$ and S ORFs, the analysis was restricted to the mammalian orthohepadnaviruses (Figure 1C,D). To assess potential long-branch effects and whether an outgroup affected tree topology, the phylogeny was also constructed using the P ORF of only the mammalian viruses (Supplementary Figure S1A), and for the $C$ and S ORFs including the bluegill virus as an outgroup (Supplementary Figure S1B,C).

\section{Discussion}

Our data indicate that Taï Forest hepadnavirus is sufficiently different from classified orthohepadnaviruses to represent a separate, likely novel taxon (Figure 1 and Figure S1). Although the precise phylogenetic relationship of the virus to other members of the genus is not clarified, as indicated by the often low statistical support of major branches (Figure 1 and Figure S1), our data add to the growing body of evidence demonstrating a vast diversity of these viruses [1-13], whose discovery and characterization will provide new insights into the origins and evolution of the hepatitis $B$ viruses [40]. The analysis of the largest and most conserved P ORF sequence indicates the Tai Forest hepadnavirus falls within a clade including most known bat and all known rodent orthohepadnaviruses (Figure 1B), though this topology was not supported when the more divergent avihepadnaviruses were excluded from the analysis (Figure S1). Further discovery and sequencing of orthohepadnaviruses from a larger diversity of mammals will likely help to resolve this phylogenetic uncertainty. 
In conclusion, we report the first orthohepadnavirus detected in a member of the order Artiodactyla, with considerable viral copy numbers in blood and several organs that are suggestive of widespread viremia. These results provide further evidence for a broad unexplored diversity of orthohepadnaviruses in mammals [41,42]. The low prevalence of the Taï Forest hepadnavirus encountered in the available sample set indicates that a spillover event from another species cannot be ruled out at this point. The scavenging behavior that has been reported for duikers might increase the likelihood for such a cross-species transmission $[43,44]$, but more research on the orthohepadnaviruses circulating in the mammalian community of Taï National Park, Côte d'Ivoire is needed to clarify this. Given our results, understanding the potential impact of orthohepadnaviruses on animal health, including livestock species, represents another important area of future research. Serological and histopathological investigations of carcasses representing a broader diversity of species will allow us to assess the extent of exposure and diversity of orthohepadnaviruses in this ecosystem, as well as potential associations of infection with disease [45]. Further investigations into the evolution of these viruses in a broader range of hosts will provide a clearer understanding of the processes (co-divergence vs. host-switching) that underlie hepadnavirus diversification.

Supplementary Materials: The following are available online at http:/ / www.mdpi.com/1999-4915/11/3/279/s1, Figure S1: Phylogenetic relationship of Taï Forest hepadnavirus to other viruses of the family Hepadnaviridae, Table S1: Reads mapping to Taï Forest hepadnavirus following enrichment by VirCapSeq-VERT, Table S2: List of duiker tissues collected during necropsies and tested for the Taï Forest hepadnavirus.; Files related to the phylogenetic analysis are available via: https:/ / github.com/jangogarten/Maxwells-duiker-hepatitis-B-virus. All reads from the two positive samples are avaialbe under SRA accession number: PRJNA526079 and the full genome has been uploaded to GenBank with the Accession Number: MK620908.

Author Contributions: Conceptualization, J.F.G., M.U., T.B., W.I.L., S.C.-S., and F.H.L.; methodology, J.F.G., M.U., T.B., N.M., W.I.L., S.C.-S., and F.H.L.; software, J.F.G., K.J., T.B., W.I.L., and S.C.-S.; validation, J.F.G., M.U., T.B., W.I.L., S.C.-S., and F.H.L.; formal analysis, J.F.G., M.U., B.L., K.J., T.B., W.I.L., S.C.-S., and F.H.L.; investigation, J.F.G., M.U., N.B., J.G., T.L., A.O., and N.M.; resources, E.C.-H., T.F.N., T.B., S.C.-S., W.I.L., and F.H.L.; data curation, J.F.G., M.U., and K.J.; writing-original draft preparation, J.F.G., S.C.-S. and F.H.L.; writing-review and editing, J.F.G., M.U., N.B., B.L., J.G., K.J., T.L., A.O., E.C.H., T.F.N., N.M., T.B., S.C.-S., W.I.L., and F.H.L.; visualization, J.F.G. and S.C.-S.; supervision, J.F.G., E.C.-H., T.F.N., T.B., S.C.-S., W.I.L., and F.H.L.; project administration, J.F.G., T.B., S.C.-S., W.I.L., and F.H.L.; funding acquisition, J.F.G., T.B., S.C.-S., W.I.L., and F.H.L.

Funding: J.F.G. was supported by the DAAD with funds from the German Federal Ministry of Education and Research (BMBF) and the People Programme (Marie Curie Actions) of the European Union's Seventh Framework Programme (FP7/2007-2013) under REA grant agreement $n^{\circ} 605728$ (P.R.I.M.E.-Postdoctoral Researchers International Mobility Experience). Core-funding for the Taï Chimpanzee Project is provided by the Max Planck Society, equipment for necropsies was provided through Deutsche Forschungsgemeinschaft (DFG: KL 2521/1-1). This research was also benefited from interactions with the DFG research group "Sociality and Health in Primates" (FOR2136). B.L., N.B., J.G., K.J., N.M., T.B., and W.I.L. were supported by grant AI109761 from the National Institutes of Health under the auspices of the Center for Research in Diagnostics and Discovery.

Acknowledgments: We thank the Ministère de l'Enseignement Supérieur et de la Recherche Scientifique and the Ministère de Eaux et Fôrests in Côte d'Ivoire, and the Office Ivoirien des Parcs et Réserves for permitting the study. We are grateful to the Centre Suisse de Recherches Scientifiques en Côte d'Ivoire and the staff members of the Taï Chimpanzee Project for their support. We are indebted to the Max Planck Society providing core funding for the Taï Chimpanzee Project since 1997. We are grateful for the necropsies performed by a courageous team of field veterinarians including: Siv Aina Leendertz, Sonja Metzger, Kamilla Pleh, and Hélène De Nys. We would like to thank the government of the Central African Republic for their long term support, especially the Ministère des Eaux, Fôrets, Chasse et Peche and the Ministère de l'Enseignement Superieur, de la Recherche Scientifique et de l'Innovation Technologique. In particular, we thank Jean-Baptiste Mamang-Kanga, Guian Zokoe and Christian Ndadet. We thank the staff of DSPA and especially those concerned with the Primate Habituation Programme, for logistical support in the field, and World Wide Fund (WWF) for their support at DSPA and in Bangui. We thank Jan Felix Drexler and Claus-Thomas Bock for constructive discussions about interpreting these results.

Conflicts of Interest: The authors declare no conflict of interest. The funders had no role in the design of the study; in the collection, analyses, or interpretation of data; in the writing of the manuscript, or in the decision to publish the results.

\section{References}

1. Lanford, R.E.; Chavez, D.; Brasky, K.M.; Burns, R.B.; Rico-Hesse, R. Isolation of a hepadnavirus from the woolly monkey, a New World primate. Proc. Natl. Acad. Sci. USA 1998, 95, 5757-5761. [CrossRef] 
2. Souza, B.F.d.C.D.; König, A.; Rasche, A.; de Oliveira Carneiro, I.; Stephan, N.; Corman, V.M.; Roppert, P.L.; Goldmann, N.; Kepper, R.; Müller, S.F. A novel hepatitis B virus species discovered in capuchin monkeys sheds new light on the evolution of primate hepadnaviruses. J. Hepatol. 2018, 68, 1114-1122.

3. Grethe, S.; Heckel, J.O.; Rietschel, W.; Hufert, F.T. Molecular epidemiology of hepatitis B virus variants in nonhuman primates. J. Virol. 2000, 74, 5377-5381. [CrossRef] [PubMed]

4. Starkman, S.; MacDonald, D.; Lewis, J.; Holmes, E.; Simmonds, P. Geographic and species association of hepatitis B virus genotypes in non-human primates. Virology 2003, 314, 381-393. [CrossRef]

5. He, B.; Zhang, F.; Xia, L.; Hu, T.; Chen, G.; Qiu, W.; Fan, Q.; Feng, Y.; Guo, H.; Tu, C. Identification of a novel Orthohepadnavirus in pomona roundleaf bats in China. Arch. Virol. 2015, 160, 335-337. [CrossRef]

6. He, B.; Fan, Q.; Yang, F.; Hu, T.; Qiu, W.; Feng, Y.; Li, Z.; Li, Y.; Zhang, F.; Guo, H. Hepatitis virus in long-fingered bats, Myanmar. Emerg. Infect. Dis. 2013, 19, 638. [CrossRef] [PubMed]

7. Wang, B.; Yang, X.L.; Li, W.; Zhu, Y.; Ge, X.Y.; Zhang, L.B.; Zhang, Y.Z.; Bock, C.T.; Shi, Z.L. Detection and genome characterization of four novel bat hepadnaviruses and a hepevirus in China. Virol. J. 2017, 14, 40. [CrossRef] [PubMed]

8. Nie, F.Y.; Lin, X.D.; Hao, Z.Y.; Chen, X.N.; Wang, Z.X.; Wang, M.R.; Wu, J.; Wang, H.W.; Zhao, G.; Ma, R.Z. Extensive diversity and evolution of hepadnaviruses in bats in China. Virology 2018, 514, 88-97. [CrossRef] [PubMed]

9. Summers, J.; Smolec, J.M.; Snyder, R. A virus similar to human hepatitis B virus associated with hepatitis and hepatoma in woodchucks. Proc. Natl. Acad. Sci. USA 1978, 75, 4533-4537. [CrossRef]

10. Testut, P.; Renard, C.A.; Terradillos, O.; Vitvitski-Trepo, L.; Tekaia, F.; Degott, C.; Blake, J.; Boyer, B.; Buendia, M.A. A new hepadnavirus endemic in arctic ground squirrels in Alaska. J. Virol. 1996, 70, 4210-4219.

11. Seeger, C.; Ganem, D.; Varmus, H.E. Nucleotide sequence of an infectious molecularly cloned genome of ground squirrel hepatitis virus. J. Virol. 1984, 51, 367-375.

12. Marion, P.L.; Oshiro, L.S.; Regnery, D.C.; Scullard, G.H.; Robinson, W.S. A virus in Beechey ground squirrels that is related to hepatitis B virus of humans. Proc. Natl. Acad. Sci. USA 1980, 77, 2941-2945. [CrossRef]

13. Aghazadeh, M.; Shi, M.; Barrs, V.R.; McLuckie, A.J.; Lindsay, S.A.; Jameson, B.; Hampson, B.; Holmes, E.C.; Beatty, J.A. A Novel Hepadnavirus Identified in an Immunocompromised Domestic Cat in Australia. Viruses 2018, 10, 269. [CrossRef]

14. World Health Organization. Disease Burden and Mortality Estimates: Cause-Specific Mortality, 2000-2016; World Health Organization: Geneva, Switzerland; Available online: https:/ /www.who.int/healthinfo/global_ burden_disease/estimates/en/ (accessed on 15 January 2019).

15. Drexler, J.F.; Geipel, A.; König, A.; Corman, V.M.; van Riel, D.; Leijten, L.M.; Bremer, C.M.; Rasche, A.; Cottontail, V.M.; Maganga, G.D. Bats carry pathogenic hepadnaviruses antigenically related to hepatitis B virus and capable of infecting human hepatocytes. Proc. Natl. Acad. Sci. USA 2013, 110, 16151-16156. [CrossRef]

16. Takahashi, K.; Brotman, B.; Usuda, S.; Mishiro, S.; Prince, A.M. Full-genome sequence analyses of hepatitis B virus (HBV) strains recovered from chimpanzees infected in the wild: Implications for an origin of HBV. Virology 2000, 267, 58-64. [CrossRef]

17. Littlejohn, M.; Locarnini, S.; Yuen, L. Origins and evolution of hepatitis B virus and hepatitis D virus. Cold Spring Harb. Perspect. Med. 2016, 6, a021360. [CrossRef]

18. Gogarten, J.F.; Akoua-Koffi, C.; Calvignac-Spencer, S.; Leendertz, S.A.J.; Weiss, S.; Couacy-Hymann, E.; Koné, I.; Peeters, M.; Wittig, R.M.; Boesch, C. The ecology of primate retroviruses-an assessment of 12 years of retroviral studies in the Taï national park area, Côte d'Ivoire. Virology 2014, 460, 147-153. [CrossRef]

19. Grützmacher, K.S.; Köndgen, S.; Keil, V.; Todd, A.; Feistner, A.; Herbinger, I.; Petrzelkova, K.; Fuh, T.; Leendertz, S.A.; Calvignac-Spencer, S. Codetection of respiratory syncytial virus in habituated wild western lowland gorillas and humans during a respiratory disease outbreak. EcoHealth 2016, 13, 499-510. [CrossRef]

20. Hoffmann, C.; Zimmermann, F.; Biek, R.; Kuehl, H.; Nowak, K.; Mundry, R.; Agbor, A.; Angedakin, S.; Arandjelovic, M.; Blankenburg, A.; et al. Persistent anthrax as a major driver of wildlife mortality in a tropical rainforest. Nature 2017, 548, 82. [CrossRef]

21. Radonić, A.; Metzger, S.; Dabrowski, P.W.; Couacy-Hymann, E.; Schuenadel, L.; Kurth, A.; Mätz-Rensing, K.; Boesch, C.; Leendertz, F.H.; Nitsche, A. Fatal monkeypox in wild-living sooty mangabey, Cote d'Ivoire, 2012. Emerg. Infect. Dis. 2014, 20, 1009. [CrossRef] 
22. Schroeder, K.; Nitsche, A. Multicolour, multiplex real-time PCR assay for the detection of human-pathogenic poxviruses. Mol. Cell. Probes 2010, 24, 110-113. [CrossRef]

23. Briese, T.; Kapoor, A.; Mishra, N.; Jain, K.; Kumar, A.; Jabado, O.J.; Lipkin, W.I. Virome capture sequencing enables sensitive viral diagnosis and comprehensive virome analysis. MBio 2015, 6, e01491-e041515. [CrossRef]

24. Bolger, A.M.; Lohse, M.; Usadel, B. Trimmomatic: A flexible trimmer for Illumina sequence data. Bioinformatics 2014, 30, 2114-2120. [CrossRef]

25. Bankevich, A.; Nurk, S.; Antipov, D.; Gurevich, A.A.; Dvorkin, M.; Kulikov, A.S.; Lesin, V.M.; Nikolenko, S.I.; Pham, S.; Prjibelski, A.D. SPAdes: A new genome assembly algorithm and its applications to single-cell sequencing. J. Comput. Biol. 2012, 19, 455-477. [CrossRef]

26. Kearse, M.; Moir, R.; Wilson, A.; Stones-Havas, S.; Cheung, M.; Sturrock, S.; Buxton, S.; Cooper, A.; Markowitz, S.; Duran, C. Geneious Basic: An integrated and extendable desktop software platform for the organization and analysis of sequence data. Bioinformatics 2012, 28, 1647-1649. [CrossRef]

27. Li, H. Aligning sequence reads, clone sequences and assembly contigs with BWA-MEM. arXiv 2013, arXiv:1303.3997.

28. Gouy, M.; Guindon, S.; Gascuel, O. SeaView version 4: A multiplatform graphical user interface for sequence alignment and phylogenetic tree building. Mol. Biol. Evol. 2009, 27, 221-224. [CrossRef]

29. Lefort, V.; Longueville, J.E.; Gascuel, O. SMS: Smart model selection in PhyML. Mol. Biol. Evol. 2017, 34, 2422-2424. [CrossRef]

30. Rambaut, A.; Lam, T.T.; Max Carvalho, L.; Pybus, O.G. Exploring the temporal structure of heterochronous sequences using TempEst (formerly Path-O-Gen). Virus Evol. 2016, 2, vew007. [CrossRef]

31. Suchard, M.A.; Lemey, P.; Baele, G.; Ayres, D.L.; Drummond, A.J.; Rambaut, A. Bayesian phylogenetic and phylodynamic data integration using BEAST 1.10. Virus Evol. 2018, 4, vey016. [CrossRef]

32. Rambaut, A.; Drummond, A.J.; Xie, D.; Baele, G.; Suchard, M.A. Posterior summarisation in Bayesian phylogenetics using Tracer 1.7. Syst. Biol. 2018, 67, 901-904. [CrossRef]

33. Drummond, A.J.; Rambaut, A. BEAST: Bayesian evolutionary analysis by sampling trees. BMC Evol. Biol. 2007, 7, 214. [CrossRef]

34. Katoh, K.; Standley, D.M. MAFFT multiple sequence alignment software version 7: Improvements in performance and usability. Mol. Biol. Evol. 2013, 30, 772-780. [CrossRef]

35. Schaefer, S.; Glebe, D.; Wend, U.C.; Oyunbileg, J.; Gerlich, W.H. Universal primers for real-time amplification of DNA from all known Orthohepadnavirus species. J. Clin. Virol. 2003, 27, 30-37. [CrossRef]

36. Mommeja-Marin, H.; Mondou, E.; Blum, M.R.; Rousseau, F. Serum HBV DNA as a marker of efficacy during therapy for chronic HBV infection: Analysis and review of the literature. Hepatology 2003, 37, 1309-1319. [CrossRef]

37. Le Guenno, B.; Formenty, P.; Wyers, M.; Gounon, P.; Walker, F.; Boesch, C. Isolation and partial characterisation of a new strain of Ebola virus. Lancet 1995, 345, 1271-1274. [CrossRef]

38. Korba, B.E.; Wells, F.; Tennant, B.; Cote, P.; Gerin, J. Lymphoid cells in the spleens of woodchuck hepatitis virus-infected woodchucks are a site of active viral replication. J. Virol. 1987, 61, 1318-1324.

39. Michalak, T.I.; Hodgson, P.D.; Churchill, N.D. Posttranscriptional inhibition of class I major histocompatibility complex presentation on hepatocytes and lymphoid cells in chronic woodchuck hepatitis virus infection. J. Virol. 2000, 74, 4483-4494. [CrossRef]

40. Rasche, A.; Souza, B.F.D.C.D.; Drexler, J.F. Bat hepadnaviruses and the origins of primate hepatitis B viruses. Curr. Opin. Virol. 2016, 16, 86-94. [CrossRef]

41. Lauber, C.; Seitz, S.; Mattei, S.; Suh, A.; Beck, J.; Herstein, J.; Börold, J.; Salzburger, W.; Kaderali, L.; Briggs, J.A. Deciphering the origin and evolution of hepatitis B viruses by means of a family of non-enveloped fish viruses. Cell Host Microbe 2017, 22, 387-399. e6. [CrossRef]

42. Dill, J.A.; Camus, A.C.; Leary, J.H.; Di Giallonardo, F.; Holmes, E.C.; Ng, T.F.F. Distinct viral lineages from fish and amphibians reveal the complex evolutionary history of hepadnaviruses. J. Virol. 2016, 90, 7920-7933. [CrossRef]

43. Leroy, E.M.; Rouquet, P.; Formenty, P.; Souquière, S.; Kilbourne, A.; Froment, J.M.; Bermejo, M.; Smit, S.; Karesh, W.; Swanepoel, R. Multiple Ebola virus transmission events and rapid decline of central African wildlife. Science 2004, 303, 387-390. [CrossRef] 
44. Rouquet, P.; Froment, J.M.; Bermejo, M.; Kilbourn, A.; Karesh, W.; Reed, P.; Kumulungui, B.; Yaba, P.; Délicat, A.; Rollin, P.E. Wild animal mortality monitoring and human Ebola outbreaks, Gabon and Republic of Congo, 2001-2003. Emerg. Infect. Dis. 2005, 11, 283. [CrossRef]

45. Zimmermann, F.; Köhler, S.M.; Nowak, K.; Dupke, S.; Barduhn, A.; Düx, A.; Lang, A.; De Nys, H.M.; Gogarten, J.F.; Grunow, R. Low antibody prevalence against Bacillus cereus biovar anthracis in Taï National Park, Côte d'Ivoire, indicates high rate of lethal infections in wildlife. PLoS Negl. Trop. Dis. 2017, 11, e0005960. [CrossRef]

(c) 2019 by the authors. Licensee MDPI, Basel, Switzerland. This article is an open access article distributed under the terms and conditions of the Creative Commons Attribution (CC BY) license (http:/ / creativecommons.org/licenses/by/4.0/). 